Published in final edited form as:

ACS Chem Neurosci. 2020 March 18; 11(6): 851-863. doi:10.1021/acschemneuro.9b00475.

\title{
Photodynamic Modification of Native HCN Channels Expressed in Thalamocortical Neurons
}

\author{
Fusheng Wei§, \\ Department of Anesthesiology, The First Affiliated Hospital of Nanchang University, Nanchang \\ 330031, Jiangxi, China; Department of Physiology and Biophysics, Department of Anatomy and \\ Neurobiology, School of Medicine, Virginia Commonwealth University, Richmond, Virginia 23284, \\ United States
}

\section{Qiang Wang§,}

Department of Physiology and Biophysics, Department of Anatomy and Neurobiology, School of Medicine, Virginia Commonwealth University, Richmond, Virginia 23284, United States

Jizhong Han§,

Department of Physiology and Biophysics, Department of Anatomy and Neurobiology, School of Medicine, Virginia Commonwealth University, Richmond, Virginia 23284, United States

\section{Priyodarshan Goswamee,}

Department of Physiology and Biophysics, Department of Anatomy and Neurobiology, School of Medicine, Virginia Commonwealth University, Richmond, Virginia 23284, United States

\section{Ankush Gupta,}

Department of Physiology and Biophysics, Department of Anatomy and Neurobiology, School of Medicine, Virginia Commonwealth University, Richmond, Virginia 23284, United States

\section{Adam Rory McQuiston,}

Department of Physiology and Biophysics, Department of Anatomy and Neurobiology, School of Medicine, Virginia Commonwealth University, Richmond, Virginia 23284, United States

\section{Qinglian Liu,}

Department of Physiology and Biophysics, Department of Anatomy and Neurobiology, School of Medicine, Virginia Commonwealth University, Richmond, Virginia 23284, United States

\section{Lei Zhou}

Department of Physiology and Biophysics, Department of Anatomy and Neurobiology, School of Medicine, Virginia Commonwealth University, Richmond, Virginia 23284, United States

\footnotetext{
Corresponding Author: Lei Zhou - Department of Physiology and Biophysics, Department of Anatomy and Neurobiology, School of Medicine, Virginia Commonwealth University, Richmond, Virginia 23284, United States, lzhou@ vcu.edu.

$\S_{\text {Author Contributions }}$

These authors contribute equally. F.W., Q.W., P.G., J.H., and A.G. performed research and analyzed data. A.R.M., Q.L., and L.Z. designed research, contributed analytic tools, and wrote the paper.

ASSOCIATED CONTENT

Supporting Information

The Supporting Information is available free of charge at https://pubs.acs.org/doi/10.1021/acschemneuro.9b00475.

Six supporting figures illustrating raw data, additional data analysis and control results (PDF)

The authors declare no competing financial interest.
} 


\section{Abstract}

The photodynamic process requires three elements: light, oxygen, and photosensitizer, and involves the formation of singlet oxygen, the molecular oxygen in excited electronic states. Previously, we reported that heterologously expressed hyperpolarization-activated cAMP-gated (HCN) channels in excised membrane patches are sensitive to photodynamic modification (PDM). Here we extend this study to native HCN channels expressed in thalamocortical (TC) neurons in the ventrobasal (VB) complex of the thalamus and dopaminergic neurons (DA) of the ventral tegmental area (VTA). To do this, we introduced the photosensitizer FITC-cAMP into TCs or DAs of rodent brain slices via a whole-cell patch-clamp recording pipette. After illumination with blue light pulses, we observed an increase in the voltage-insensitive, instantaneous $I_{\text {inst }}$ component, accompanied by a long-lasting decrease in the hyperpolarization-dependent $I_{\mathrm{h}}$ component. Both $I_{\mathrm{h}}$ and the increased $I_{\text {inst }}$ after PDM could be blocked by the HCN blockers $\mathrm{Cs}^{+}$and ZD7288. When FITC and cAMP were dissociated and loaded into neurons as two separate chemicals, light application did not result in any long-lasting changes of the $\mathrm{HCN}$ currents. In contrast, light pulses applied to HCN2-/- neurons loaded with FITC-cAMP generated a much greater reduction in the $I_{\text {inst }}$ component compared to that of WT neurons. Next, we investigated the impact of the longlasting increases in $I_{\text {inst }}$ after PDM on the cellular physiology of VB neurons. Consistent with an upregulation of $\mathrm{HCN}$ channel function, PDM elicited a depolarization of the resting membrane potential (RMP). Importantly, Trolox-C, an effective quencher for singlet oxygen, could block the PDM-dependent increase in $I_{\text {inst }}$ and depolarization of the RMP. We propose that PDM of native HCN channels under physiological conditions may provide a photodynamic approach to alleviate $\mathrm{HCN}$ channelopathy in certain pathological conditions.

\section{Graphical Abstract}



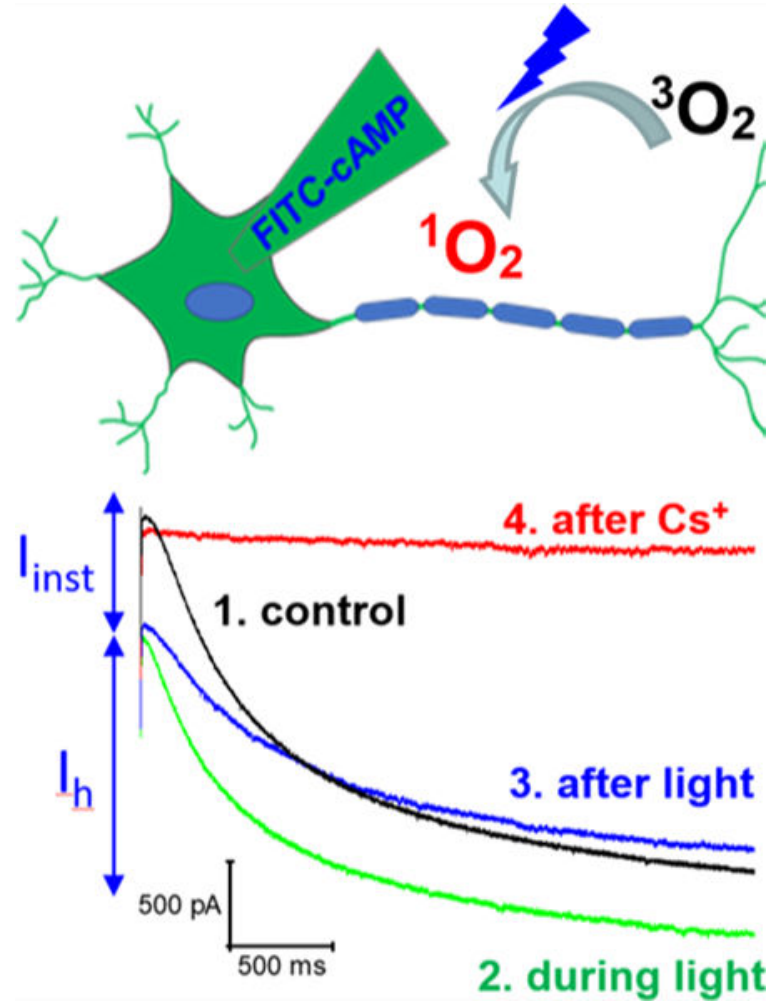

\section{Keywords}

Photodynamic modification; photosensitizer; $\mathrm{HCN}$ channel; $\mathrm{I}_{\mathrm{h}}$ current; thalamocortical neuron; ventral tegmental area

\section{INTRODUCTION}

Activation of hyperpolarization-activated cAMP-gated $(\mathrm{HCN})$ channels produce the hyperpolarization-activated $I_{\mathrm{h}}$ (or $I_{\mathrm{f}}$, funny current) current that was originally observed in neurons and cardiac cells. ${ }^{1,2}$ Importantly, $\mathrm{HCN}$ channels contribute to cardiac pacemaking, sensation of pain, and learning and memory. ${ }^{1-5}$ Compared to other types of ion channels, $\mathrm{HCN}$ channels show unique ion channel properties. HCN channels activate upon membrane hyperpolarization, have mixed permeability for both $\mathrm{K}^{+}$and $\mathrm{Na}^{+}$ions, have a reversal potential near $-30 \mathrm{mV}$, and respond directly to binding by intracellular cAMP. Four mammalian isoforms exist, $\mathrm{HCN} 1$ to $\mathrm{HCN} 4$, as well as an isoform from sea urchin, spHCN. 4-6 At the molecular level, each functional $\mathrm{HCN}$ channel contains four subunits. Each subunit consists of a six-helix transmembrane domain that is homologous to that of the voltage-gated $\mathrm{K}$ channels and a canonical cyclic-nucleotide binding domain in the $\mathrm{C}$ terminus. Thus, $\mathrm{HCN}$ channels contain the machineries necessary for both voltagedependent and ligand-dependent gating processes.

The RNA transcripts of $H C N 1-4$ have been broadly identified in the heart and brain. ${ }^{7,8}$ $\mathrm{HCN} 2$ and $\mathrm{HCN} 4$ are the two major isoforms expressed in the sinoatrial (SA), atrioventricular (AV) nodes, and Purkinje fibers of the heart, which are the regions 
responsible for the initiation and conduction of cardiac action potentials (AP). ${ }^{9,10} \mathrm{HCN}$ channels are also broadly expressed in the brain where they contribute to the resting membrane potential (RMP), dendritic integration of synaptic inputs, and the generation and propagation of AP. ${ }^{11-14}$ At the cellular level, activation of HCN channels depolarizes the membrane potential and reduces the input resistance. Conversely, downregulation of HCN channel function, through genetic or pharmacological mechanisms, leads to a hyperpolarization of the RMP, an increase in cell input resistance $\left(R_{\mathrm{in}}\right)$, altered dendritic integration of synaptic inputs, and changes in the shape and propagation of AP. ${ }^{15}$ At the behavioral level, HCN transgenic or knockout mice show cardiac arrythmia, defects in learning and memory, impaired motor performance, and reduced sensation of pain. ${ }^{16-18}$

In our previous study of the state-dependent interactions between cAMP and the full-length mHCN2 channel, we applied patch-clamp fluorometry and used different fluorescently labeled cAMP molecules to identify cAMP binding. ${ }^{19,20}$ Through studying heterologously expressed HCN channels in excised membrane patches, we discovered that laser illumination of FITC-cAMP bound to the mHCN2 channel slowed down channel deactivation and increased the voltage-insensitive instantaneous $\left(I_{\text {inst }}\right)$ component. ${ }^{21,22}$ In both mouse $\mathrm{HCN} 2$ and spHCN channels, we found that photodynamic modification effectively promotes the activation of $I_{\text {inst }}$. We subsequently identified that singlet oxygen $\left({ }^{1} \mathrm{O}_{2}\right)$, the molecular oxygen in high electronic energy states, was the major mechanism in the photochemical process.

$I_{\text {inst }}$ is the conductance carried by $\mathrm{HCN}$ channels that displays an immediate current response to a change in membrane potential and is independent of the time-dependent, delayed increase in current amplitude associated with the voltage-dependent gating process. Being intrinsically insensitive to voltage, $I_{\text {inst }}$ is constitutively conductive regardless of the membrane potential and therefore may have distinct significant physiological contributions. Interestingly, the existence of $I_{\text {inst }}$ might explain the dilemma that HCN2 and HCN4 carry important physiological functions, but their activation requires nonphysiological negative potentials ( $-100 \mathrm{mV}$ or more negative potentials). In both native and heterologous expression systems, the $I_{\text {inst }}$ carried by HCN channels has been identified, but the corresponding molecular basis underlying the decoupling between the gate and the voltage sensor has been unclear. ${ }^{23-25}$

$\mathrm{HCN}$ channelopathy underlies a number of neurological disorders including recently identified links to autism spectrum disorders (ASD). The potential involvement of $\mathrm{HCN}$ in ASD may be functionally tied to the protein Shank3, a scaffold protein that is highly enriched in the post-synaptic density. ${ }^{26,27}$ Shank3 knockout mice show key features of ASD including repetitive behavior and deficits in social interaction. ${ }^{28-30}$ Importantly, Shank3deficient hippocampal neurons displayed reduced $I_{\mathrm{h}}$ current, which resulted in increased $R_{\text {in }}$ and decreased RMP. ${ }^{31-33}$ Both Shank3 and HCN2 are expressed at high levels in the thalamus. Although the thalamus plays a crucial role in processing sensory information and structural and functional abnormalities have been identified in the thalamus of ASD patients, less is known regarding how the thalamus contributes to ASD symptoms at the cellular level. 34-36 We have characterized the $I_{\mathrm{h}}$ current and the cell physiology of TC neurons from WT, Shank3-/-, and HCN2-I- mutant mice. Indeed, Shank3 deficiency led to a reduction in 
$\mathrm{HCN}$ expression and produced a similar trend of changes in RMP, $R_{\mathrm{in}}$, and AP firing between Shank3-/- and HCN2-/- neurons. ${ }^{33}$

Here we extend our previous investigations of heterologously expressed $\mathrm{HCN}$ channels to native HCN channels expressed in VB and VTA neurons. We found that PDM with FITCcAMP as the photosensitizer resulted in a long-lasting increase in $I_{\text {inst }}$ and a corresponding decrease in $I_{\mathrm{h}}$. Furthermore, we observed a significant depolarization in membrane potential suggesting that the PDM-augmented $I_{\text {inst }}$ could partially compensate for the HCN channelopathy such as observed in Shank3-deficient neurons.

\section{RESULTS}

\section{Photodynamic Modification of Native HCN Channels in Wild-Type Thalamocortical Neurons.}

Wild-type (WT) VB neurons were loaded with either FITC-cAMP (20 $\mu \mathrm{M}$; Figure 1A) or FITC (20 $\mu \mathrm{M}$; Figure 1B) through the whole-cell recording pipette. To generate $I_{\mathrm{h}}$ current in VB TCs, a hyperpolarizing voltage step from -40 to $-100 \mathrm{mV}$ was delivered through the recording pipette. To examine potential PDM, light pulses $(6 \mathrm{~s})$ were delivered to the cell through the objective lens. Upon the first light exposure, immediate increases in $I_{\mathrm{h}}$ current amplitude and the rate of channel activation were observed (Figures S1-S2 and 1C,D). The enhancement of $I_{\mathrm{h}}$ current was reversible and immediately returned to a lower level after light illumination was discontinued. However, continued light illumination led to a gradual increase in the $I_{\text {inst }}$ component that remained at an elevated level even following discontinuation of light exposure. This augmentation of $I_{\text {inst }}$ (in absolute value) was more significant in cells loaded with FITC-cAMP than cells loaded with FITC (Figure 1A,B,E). Thus, with FITC-cAMP in the cell, the long-lasting increase in the $I_{\text {inst }}$ component after PDM was similar to our previous observations with recombinant HCN2 channels which confirmed that FITC-cAMP can be used as a site-specific photosensitizer in PDM of HCN channels. ${ }^{19,21}$

\section{Increased $l_{\text {inst }}$ after PDM Was Blocked by the HCN Blockers $\mathrm{Cs}^{+}$and ZD7288.}

To confirm the increased $I_{\text {inst }}$ is carried by HCN channels, we applied Cs ${ }^{+}$or ZD7288 to the bath solution. $\mathrm{Cs}^{+}$in millimolar range can effectively block the $\mathrm{HCN}$ channel from the extracellular side through blocking the ion conducting pore. Indeed, the $I_{\mathrm{h}}$ component could be blocked within 1-2 min after $\mathrm{Cs}^{+}$application (1 mM; Figure 1C). Furthermore, extracellular $\mathrm{Cs}^{+}$also blocked the $I_{\text {inst }}$ with FITC-cAMP in the pipette solution after PDM (Figure 1E). In contrast, $\mathrm{Cs}^{+}$had little effect on the increases in $I_{\text {inst }}$ after PDM in the presence of FITC alone (Figure 1F). Because FITC is a nonspecific photosensitizer inside the cell, it is possible that PDM with FITC alone led to nonspecific increases in other types of conductance.

ZD7288 is a more selective blocker than $\mathrm{Cs}^{+}$for $\mathrm{HCN}$ channels. However, ZD7288 must diffuse through the cell membrane to bind to the intracellular vestibule of the ion conducting pore to block HCN channels. Therefore, blockade of the $I_{\mathrm{h}}$ component took more than $5 \mathrm{~min}$ when ZD7288 (50 $\mu \mathrm{M})$ was applied by bath (Figure 2A,B). ZD7288 application also 
increased the time constant for voltage-dependent channel activation (Figure 2C).

Importantly, ZD7288 effectively blocked the $I_{\text {inst }}$ component within $\sim 3$ min of application, at a time scale slightly faster than the block of $I_{\mathrm{h}}$ (Figure 2D,E).

To further confirm the effect is specific to FITC-cAMP, we added cAMP alone (20 $\mu \mathrm{M})$ to the pipette solution and applied the same protocol of light pulses. With cAMP in the pipette solution, light pulses transiently increased the amplitude and the opening kinetics of the $I_{\mathrm{h}}$ component, which immediately returned to control level after light pulses and could be blocked effectively by bath applied $\mathrm{Cs}^{+}$(Figure 3A-C). However, light application did not lead to long-lasting increases in the $I_{\text {inst }}$ component (Figures 3D,E and S3).

$\mathrm{HCN} 2$ is the major $\mathrm{HCN}$ subunit expressed in thalamocortical neurons and accounts for the largest fraction of isolated $I_{\mathrm{h}}$ currents. ${ }^{33,37} \mathrm{Next}$, we investigated PDM of native HCN channels in HCN2-I- VB neurons. As expected, the $I_{\mathrm{h}}$ currents isolated from HCN2-/neurons were smaller in amplitude, had slower opening kinetics, and were likely mediated by $\mathrm{HCN} 4{ }^{8,38,39}$ (Figure 4A,B). Light illumination applied to HCN2-/- neurons loaded with FITC-cAMP led to little changes in the amplitude and opening kinetics of the $I_{\mathrm{h}}$ current. After light, we observed a decrease in $I_{\mathrm{h}}$ amplitude of HCN2-/- neurons that after normalization was comparable to that observed in WT neurons (Figure 4C,D). However, the sustained increase in $I_{\text {inst }}$ following the termination of light illumination was much smaller in HCN2-/- neurons (Figure 4E,F). These results suggest that HCN2 channels were probably more sensitive to PDM than other $\mathrm{HCN}$ channels expressed in VB neurons.

\section{PDM Does Not Shift the $V_{1 / 2}$ to Increase $l_{\text {inst }}$.}

We next examined whether the PDM increase in $I_{\text {inst }}$ was due to a depolarizing shift in the activation range of $\mathrm{HCN}$ channels. To do this, we measured the voltage-dependent activation curve by applying a series of hyperpolarizing potentials to activate the channel to different extents and then recorded tail currents at $-130 \mathrm{mV}$ (Figure 5A). To increase the stability of whole-cell recording, we reduced the light intensity to $75 \%$ of the control level and repeated the $I-V$ measurement after light exposure (Figure 5B).

With FITC-cAMP in the pipette solution, illumination of neurons did not lead to any significant shifts in voltage-dependent channel activation. The values of $V_{1 / 2}$ (the voltage at which channel opening reaches half maximal) were $-79.6 \pm 2.3 \mathrm{mV}$ (before light) and -81.0 $\pm 2.5 \mathrm{mV}$ (after light) ( $N=12$; statistically no difference) (Figure 5C-E). To measure the kinetics of channel activation, we fit the current traces in response to hyperpolarizing voltage steps by a single-exponential function. PDM slightly increased the time constant of channel activation and made the channel open slightly faster (Figure 5F). These results suggest that the increase in $I_{\text {inst }}$ after PDM is not due to a depolarization shift in the voltage-dependent activation curve.

\section{PDM of Native HCN Channels Resulted in a Depolarization of the RMP.}

We next examined whether PDM of $I_{\text {inst }}$ had any impact on cellular physiology. HCN channels are known to contribute to the resting membrane potential and input resistance of neurons. ${ }^{1,40}$ Down-regulation of HCN channel function or expression leads to a hyperpolarization of the RMP and an increase in $R_{\mathrm{in}}$. Thus, we recorded the RMP and $R_{\mathrm{in}}$ of 
neurons loaded with $20 \mu \mathrm{M}$ FITC-cAMP ( $R_{\text {in }}$ was measured by a pulse of $+10 \mathrm{pA}$ current injection every $30 \mathrm{~s}$ ). Indeed, after light exposure (100\% light intensity), we observed a depolarization of the RMP (before, $-58.8 \pm 0.6 \mathrm{mV}$; after light, $-55.6 \pm 0.5 \mathrm{mV} ; N=5$ ) that was likely due to $I_{\text {inst }}$ driving the RMP toward its reversal potential of $-30 \mathrm{mV}$ (Figure 6A). Although, the contribution by nonselective or leakage components cannot be completely excluded. To examine this further, we loaded neurons with FITC and cAMP as two separate chemicals but did not observe any significant change in RMP under otherwise identical experimental conditions (Figure 6B). Surprisingly, for both FITC-cAMP and FITC + cAMP loaded neurons, light exposure did not lead to any significant changes in $R_{\text {in }}$ (Figure 6C,D).

To further determine if PDM of HCN channels contributed to changes in cellular physiology, we applied a different protocol that involved measuring the RMP before a $-100 \mathrm{pA}$ current injection was delivered every $30 \mathrm{~s}$. This protocol increased the magnitude of the hyperpolarization and therefore led to more complete activation of $\mathrm{HCN}$ channels. After light exposure, we observed a similar depolarizing shift in RMP in FITC-cAMP loaded neurons (Figure 6E). For $R_{\text {in }}$, we observed a decrease using the new protocol when FITCcAMP but not FITC + cAMP was included in the patch pipette (before, $172.4 \pm 17.3 \mathrm{M} \Omega$; after light, $131.8 \pm 12.6 \mathrm{M} \Omega ; N=6$; Figure $6 \mathrm{~F}$ ). It is possible that the extent of activation of $\mathrm{HCN}$ channels may contribute to the magnitude of PDM.

\section{PDM of $l_{\text {inst }}$ Was Antagonized by Trolox-C.}

Our previous study identified ${ }^{1} \mathrm{O}_{2}$ as the major mechanism mediating PDM of recombinant $\mathrm{HCN}$ channels. Those studies were carried out using excised membrane patches that permitted control of the solutions on each side of the membrane. Furthermore, excised patches eliminated contributions by complex intracellular signaling pathways. In contrast, experiments using neurons in intact brain slices were complicated by the inability to accurately control solutions and the potential contribution of intracellular signaling cascades. This presented a technical challenge in pinpointing the involvement of ${ }^{1} \mathrm{O}_{2}$ in neurons of brain slices. To this end, we tested the effects of Trolox-C, an effective quencher for ${ }^{1} \mathrm{O}_{2}$. With $10 \mathrm{mM}$ Trolox-C added to the pipette solution and $2.5 \mathrm{mM}$ Trolox-C to the bath solution, light exposure to the cells loaded with FITC-cAMP still increased the $I_{\mathrm{h}}$ amplitude and decreased the time constant for activation (Figure 7A-D). However, after the application of laser pulses, the increase in the $I_{\text {inst }}$ component was minimal and the increase in $I_{\mathrm{h}}$ amplitude was reversed (to a lower level likely due to rundown) (Figure 7E,F). Furthermore, we studied the effect of PDM on RMP under the current-clamp mode. RMP and $R_{\text {in }}$ were measured every $30 \mathrm{~s}$ by $\mathrm{a}+10 \mathrm{pA}$ current injection protocol. With Trolox-C added to the solutions, light exposure did not lead to significant changes in RMP and $R_{\text {in }}$ (Figure $7 \mathrm{G}, \mathrm{H}$ ).

\section{PDM of Native HCN Channels Expressed in VTA Dopaminergic Neurons.}

Finally, we expanded our study of PDM to HCN channels expressed in other brain areas. We chose dopaminergic neurons in the VTA that are known to express significant amount of $I_{\mathrm{h}}$ current. To validate our results of thalamocortical neurons, we carried out the experiments on VTA neurons using a separate electrophysiology setup. We loaded VTA neurons with FITC-cAMP and illuminated neurons with blue light utilizing a LED light. To improve stability of recording, the light intensity was reduced to $70 \%$ of the maximum intensity using 
a potentiometer. Similar to the results of VB neurons, we observed that exposure to blue light results in long-lasting increases in $I_{\text {inst }}$ amplitude in cells loaded with FITC-cAMP, whereas no long-lasting increased in $I_{\text {inst }}$ was seen in cAMP loaded neurons (Figure 8). Extracellularly applied Cs ${ }^{+}$blocked both $I_{\mathrm{h}}$ and $I_{\text {inst }}$ of VTA neurons after PDM. However, in contrast to the results with VB neurons, application of light pulse to VTA neurons did not consistently change the amplitude of $I_{\mathrm{h}}$. Notably, the light intensity used for the VTA setup was $\sim 3$ times higher than that used for the TC neuron setup ( $\left.780 \mathrm{vs} 260 \mathrm{~mW} / \mathrm{cm}^{2}\right)$.

\section{DISCUSSION}

Here we expand our previous study on PDM of heterologously expressed $\mathrm{HCN}$ channels to native $\mathrm{HCN}$ channels in thalamocortical and VTA neurons. A photosensitizer that specifically binds to HCN channels, FITC-cAMP, was loaded into recorded neurons through the whole-cell patch-clamp recording pipette. After the application of light pulses, we observed a long-lasting increase in $I_{\text {inst }}$ and a corresponding decrease in $I_{\mathrm{h}}$ amplitude. These observations were consistent with our previous studies on PDM of recombinant HCN channels in excised membrane patches. At the cellular level, the process of PDM resulted in significant depolarizations of the RMP of thalamocortical neurons. Finally, we confirmed that application of Trolox-C, a quencher for singlet oxygen, abolished increases in $I_{\text {inst }}$ and depolarizations of the RMP.

HCN channels were not the only protein capable of binding FITC-cAMP when FITC-cAMP was introduced into the intracellular compartment. Other proteins, including protein kinase A, cyclic-nucleotide gated ion channels, and cAMP-regulated guanine nucleotide exchange factor I, will also interact with FITC-cAMP and could potentially be modified by the process of PDM. Therefore, it is important to clarify whether our observations resulted from changes in the function of $\mathrm{HCN}$ or other channels. The observations that $\mathrm{Cs}^{+}, \mathrm{ZD} 7288$, and the genetic deletion of HCN2 in VB neurons blocked PDM suggest that the modification of $\mathrm{HCN}$ channels was the primary mechanism contributing to the increase in $I_{\text {inst }}$. Moreover, the observation that when PDM produced an increase in $I_{\text {inst }}$, a simultaneous decrease in $I_{\mathrm{h}}$ amplitudes was observed, could be explained by a shift of the population of HCN channels from conducting $I_{\mathrm{h}}$ to conducting $I_{\text {inst }}$.

Notably, light illumination resulted in a significant increase in $I_{\mathrm{h}}$ amplitude and opening kinetics, especially under the condition of cAMP alone or regular pipette solution or the first trace with FITC-cAMP or FITC. Because it is reversible and largely independent from the pipette solution, we believe that the light-dependent increase in $I_{\mathrm{h}}$ is of a different molecular nature from the photochemical modification of $\mathrm{HCN}$ channels that results in an irreversible increase in $I_{\text {inst }}$. Two chemical factors are known to be able to dynamically modulate the HCN channel opening, PIP2 and cAMP. To test the possibility of transient increases in PIP2 and cAMP upon light illumination, we added saturating concentration of PIP2 $(47 \mu \mathrm{M})$ and/or cAMP $(1 \mathrm{mM})$ into the pipette solution. However, light exposure still enhances the $I_{\mathrm{h}}$ component (Figures S4-S5). Is it possible that the reversible increase in $I_{\mathrm{h}}$ was due to a local increase in temperature? To this end, we used the glass recording pipette as a temperature sensor. We first calibrated the relationship between current (resistance) and temperature (Figure S6). Then we mounted the same electrode inside a brain slice and determined the 
increase in temperature to be around $1.1^{\circ} \mathrm{C}$. For $\mathrm{HCN}$ channels, the value of $Q_{10}$ (folds of increase in kinetics for every $10{ }^{\circ} \mathrm{C}$ ) has been reported to be $\sim 3^{41}$ or $4.5 .^{42}$ Moreover, a recent study confirmed that a temperature increase will positively shift the voltagedependent channel activation and therefore increase channel openings. ${ }^{43}$ Therefore, temperature increases could potentially provide an explanation for the transient and reversible increases in $I_{\mathrm{h}}$ activation kinetics and amplitude upon light illumination. Further studies are required to clarify the detail.

We propose that PDM of native $\mathrm{HCN}$ channels could be developed into a mechanism to compensate HCN channelopathy and reverse the related detrimental effects on cellular physiology. $\mathrm{HCN}$ channelopathy has been linked to several psychiatric disorders, including depression, anxiety, epilepsy, and autism. Due to the mixed permeability of $\mathrm{K}^{+}$and $\mathrm{Na}^{+}$ ions, the reversible potential of $\mathrm{HCN}$ channel is around $-30 \mathrm{mV}$. Reducing the expression and function of $\mathrm{HCN}$ channels, either through pharmacological (HCN blockers) or genetics (mutations or deletions of $\mathrm{HCN}$ or other regulatory genes), will lead to a hyperpolarizing shift in RMP and an increase in $R_{\mathrm{in}}$. As shown by our study, PDM of native HCN channels increases the $I_{\text {inst }}$ component and positively shifts RMP. Thus, PDM of HCN channels could be further developed as a photonic approach to compensate the abnormalities in neuronal physiology due to down regulation of HCN channels. To further expand the application potential of PDM of HCN channels, more effective photosensitizers specifically targeting a particular type of HCN subunit need to be explored.

$\mathrm{HCN}$ conductance directly contributes to neuronal input resistance. However, our results show that the impact of PDM on input resistance was small. In contrast, PDM resulted in a significant depolarization of the RMP. It is possible that the contribution to RMP by HCN channels is more significant than their contribution to $R_{\mathrm{in}}$. Moreover, there might be a mismatch between the subcellular distributions of HCN channel and FITC-cAMP within the neuron. HCN2 and HCN4 channels are known to be distributed in not only the cell body but also proximal dendrites, where the perfusion of FITC-cAMP from the soma should be limited. ${ }^{44}$ It is possible that the channels that were not modified during PDM played more significant roles in modulating the cell input resistance. Thus, more efficient delivery of photosensitizers specifically targeting HCN channels and effective PDM procedures are probably needed to generate more substantial increases in $I_{\text {inst }}$ and thus to decrease the abnormally high $R_{\text {in }}$ reported for certain psychiatric disorders.

In summary, we used intracellularly loaded FITC-cAMP as the photosensitizer and studied PDM of native HCN channels in thalamocortical neurons and VTA neurons. The amplitude and gating kinetics of the $I_{\mathrm{h}}$ current transiently increased in response to light but eventually returned to normal or a lower level after the light was turned off. The increased $I_{\text {inst }}$ was long-lasting and could be blocked by HCN blockers including $\mathrm{Cs}^{+}$and ZD7288 or genetic deletion of HCN2. These observations were consistent with our previous study of PDM of recombinant $\mathrm{HCN}$ channels which identified singlet oxygen as the major player. The $I_{\text {inst }}$ conducted by HCN channels could be further explored as a compensatory approach for HCN channelopathy such as those observed in Shank3-deficient neurons. 


\section{METHODS}

\section{Ethical Approval.}

All animal experiments were approved by the IACUC Committee of Virginia

Commonwealth University. C57BL/6J mice were used in the current study. The number of animals and procedures involving pain were minimized in compliance with regulations. Animals were subjected to regular veterinary care on a routine basis. The services of the Center for Research Animal Resources were available $24 \mathrm{~h}$ per day for any case in which veterinary care or consultation was required. Mice were housed in a standard $12 \mathrm{~h}$ light/dark cycle, with free access to food and water.

\section{HCN2 Knockout Mice.}

The brain-specific HCN2 knockout mice were generated by crossing the Nestin-Cre mice (B6.Cg-Tg(Nes-Cre)1Kln/J; stock no: 003771; Jackson Laboratory) with Floxed-HCN2 mice (exons 3-4 floxed; kindly provided by Dr. Peter McNaughton from King's College, London). ${ }^{18}$

\section{Acute Brain Slice Preparation and Whole-Cell Patch-Clamp Recording of Thalamocortical Neurons.}

Coronal thalamocortical brain slices were prepared from young mice 3-4 weeks old of either sex. In brief, mice were deeply anesthetized with isoflurane and tribromoethanol (250 $\mathrm{mg} / \mathrm{kg}$ ) and then perfused through the ascending aorta with preoxygenated $\left(95 \% \mathrm{O}_{2}\right.$ and $5 \%$ $\mathrm{CO}_{2}$ ) and ice-cold sucrose-based artificial cerebrospinal fluid (sucrose-ACSF), which contains (in mM): 240 sucrose, $2.5 \mathrm{KCl}, 1.25 \mathrm{NaH}_{2} \mathrm{PO}_{4}, 0.5 \mathrm{CaCl}_{2}, 3.5 \mathrm{MgCl}_{2}, 25$ $\mathrm{NaHCO}_{3}, 0.4$ ascorbic acid, 2 sodium pyruvate. Coronal slices of $300 \mu \mathrm{m}$ thickness were prepared using a vibratome (7550 PSDS, Campden). Slices were incubated at $32{ }^{\circ} \mathrm{C}$ for 30 min in standard ACSF (in mM: $124 \mathrm{NaCl}, 3.6 \mathrm{KCl}, 1.2 \mathrm{NaH}_{2} \mathrm{PO}_{4}, 2.5 \mathrm{CaCl}_{2}, 1.2 \mathrm{MgCl}_{2}, 25$ $\mathrm{NaHCO}_{3}, 11$ glucose, 0.4 ascorbic acid, 2 sodium pyruvate) and subsequently kept at room temperature for at least another $30 \mathrm{~min}$ prior to recording.

Brain slices were perfused continuously in the recording chamber with prewarmed carbonated standard ACSF at a rate of $2-4 \mathrm{~mL} / \mathrm{min}$ to approach the temperature around 32 ${ }^{\circ} \mathrm{C}$. Visually identified healthy neurons were recorded with glass pipettes (3-6 M $\Omega$ ) filled with internal solution (in $\mathrm{mM}$ : $130 \mathrm{~K}$-gluconate, $5 \mathrm{KCl}, 10$ phosphocreatine, $10 \mathrm{HEPES}, 0.5$ EGTA, $2 \mathrm{Na}_{2}$-ATP, 0.3 Na-GTP, $2 \mathrm{MgSO}_{4}$, pH 7.20-7.30, $290 \mathrm{mOsm}$ ). The bath solution contains: $124 \mathrm{NaCl}, 3.6 \mathrm{KCl}, 1.2 \mathrm{NaH}_{2} \mathrm{PO}_{4}, 2.5 \mathrm{CaCl}_{2}, 1.2 \mathrm{MgCl}_{2}, 25 \mathrm{NaHCO}_{3}, 11$ glucose (in $\mathrm{mM}$ ). Liquid junction potential was not corrected in this study. Neurons with the RMP more negative than $-50 \mathrm{mV}$ and the peak of action potential (AP) above $0 \mathrm{mV}$ were selected for analysis.

Input resistance $\left(R_{\mathrm{in}}\right)$ was measured at RMP based on the change in membrane potential in response to a $10 \mathrm{pA}$ current injection $(500 \mathrm{~ms})$. AP were elicited with a series of $1 \mathrm{~s}$ depolarizing current pulses at RMP. A cocktail of blockers ( $1 \mathrm{mM} \mathrm{BaCl}_{2}, 1 \mathrm{mM}$ 4-AP, 0.1 $\mathrm{mM} \mathrm{NiCl}_{2}$, and $1 \mu \mathrm{M}$ TTX) were added to the standard ACSF for the isolation of native $I_{\mathrm{h}}$ current. All recordings were obtained using a Multiclamp 700A amplifier (Molecular 
Device) and the WinWCP program (courtesy of Dr. J Dempster, Strathclyde University, Glasgow, Scotland). Measurements of $I_{\mathrm{h}}$ amplitude, $I_{\mathrm{h}}$ activation time constants, tonic, and burst firing properties were performed using Clampfit 10.7 (Molecular Device). The $I_{\text {inst }}$ amplitude corresponds to the absolute value of current measured immediately after the voltage step from -40 to $-120 \mathrm{mV}$. The $I_{\mathrm{h}}$ amplitude corresponds to the difference between the current amplitude measured at the beginning of $-120 \mathrm{mV}$ step and the value measured near the end of $-120 \mathrm{mV}$ step (Figure 1A,B).

\section{Preparation of Brain Slice and Whole-Cell Recording of VTA Neurons.}

Horizontal ventral midbrain slices containing the ventral tegmental area were prepared from 3- to 4-week old mice, as previously described. ${ }^{45}$ Briefly, mice were deeply anesthetized using isofluorane and trans-cardially perfused with sucrose-ACSF as described above. Following decapitation, brains were rapidly removed, and horizontal slices 200 to $240 \mu \mathrm{m}$ in thickness were prepared using a Leica VT1200 vibratome (Leica Microsystems, Buffalo Grove, IL, USA).

The terminal nucleus of the accessory optic tract (MT) was utilized as a landmark for the identification of putative dopaminergic cell bodies in VTA. Neurons residing $50 \mu \mathrm{m}$ from the medial side of the MT, a region known to be rich in dopaminergic cells, were utilized for whole-cell patch-clamp experiments (Figure 8A). ${ }^{45-47}$ The VTA dopaminergic cells were further identified based on stereotypical physiology features, including pacemaker-like firing in cell-attached recording mode, broad action potential with the half width longer than 1.2 $\mathrm{ms}$, large amplitude $I_{\mathrm{h}}$ currents, and a prominent "sag" in membrane potential (slow depolarization after initial hyperpolarization reaching peak level) in response to hyperpolarizing current injections. The recording glass pipette (2-5 M $\Omega$ ) was filled with internal solution (in mM: $\mathrm{KMeSO}_{4}$ 145, $\mathrm{NaCl}$ 8, Mg-ATP 2, Na-GTP 0.1, HEPES 10, EGTA 0.1). Membrane potentials or $I_{\mathrm{h}}$ currents were measured with a Model 2400 patch clamp amplifier (A-M Systems, Port Angeles, WA) and converted into a digital signal by a PCI-6040E A/D board (National Instruments, Austin, TX). The WinWCP program was used to collect and store electrophysiology data on a PC computer. For all voltage clamp experiments, series resistance was compensated to about $80 \%$, and experiments in which the access changed by more than $20 \%$ were discarded.

\section{Light Source Used for Brain Slice Recording Setup.}

For the setup used for thalamocortical neuron recordings, an LED (Thorlabs M455L3; 455 $\mathrm{nm} ; 900 \mathrm{~mW}$ ) was used for excitation. The excitation light was passed through a bandpass filter (XF3002; 450DF65), a dichroic mirror, 40× water immersion lens (Nikon; NIR APO; $40 \times / 0.80 \mathrm{~W}$; DIC N2), before reaching the brain slice in the recording chamber. The light intensity measured after the objective lens was $\sim 260 \mathrm{~mW} / \mathrm{cm}^{2}$.

For the setup used for VTA neuron recordings, a LED light source (UHP-microscopeLED-460, Prizmatix Modiin-Ilite, Givat Shmuel, Israel; $5600 \mathrm{~mW} ; 460 \mathrm{~nm}$ ) was used for excitation. The light intensity measured after the objective lens was $\sim 780 \mathrm{~mW} / \mathrm{cm}^{2}$. 


\section{Supplementary Material}

Refer to Web version on PubMed Central for supplementary material.

\section{ACKNOWLEDGMENTS}

This work was supported by National Institute of Health (NIH) grants R01GM098592 (Q.L.), R01GM109193 (L.Z. and Q.L.), R21AG055073 (A.R.M), and R01MH107507 (A.R.M.) and startup funds from Virginia Commonwealth University to Q.L. and L.Z.

\section{REFERENCES}

(1). Biel M, Wahl-Schott C, Michalakis S, and Zong X (2009) Hyperpolarization-activated cation channels: from genes to function. Physiol. Rev 89 (3), 847-85. [PubMed: 19584315]

(2). Robinson RB, and Siegelbaum SA (2003) Hyperpolarization-activated cation currents: from molecules to physiological function. Annu. Rev. Physiol 65, 453-80. [PubMed: 12471170]

(3). Stevens DR, Seifert R, Bufe B, Muller F, Kremmer E, Gauss R, Meyerhof W, Kaupp UB, and Lindemann B (2001) Hyperpolarization-activated channels HCN1 and HCN4 mediate responses to sour stimuli. Nature 413 (6856), 631-5. [PubMed: 11675786]

(4). Santoro B, Liu DT, Yao H, Bartsch D, Kandel ER, Siegelbaum SA, and Tibbs GR (1998) Identification of a gene encoding a hyperpolarization-activated pacemaker channel of brain. Cell 93 (5), 717-29. [PubMed: 9630217]

(5). Ludwig A, Zong X, Jeglitsch M, Hofmann F, and Biel M (1998) A family of hyperpolarizationactivated mammalian cation channels. Nature 393 (6685), 587-91. [PubMed: 9634236]

(6). Gauss R, Seifert R, and Kaupp UB (1998) Molecular identification of a hyperpolarizationactivated channel in sea urchin sperm. Nature 393 (6685), 583-7. [PubMed: 9634235]

(7). Moosmang S, Biel M, Hofmann F, and Ludwig A (1999) Differential distribution of four hyperpolarization-activated cation channels in mouse brain. Biol. Chem 380 (7-8), 975-80. [PubMed: 10494850]

(8). Monteggia LM, Eisch AJ, Tang MD, Kaczmarek LK, and Nestler EJ (2000) Cloning and localization of the hyperpolarization-activated cyclic nucleotide-gated channel family in rat brain. Mol. Brain Res 81 (1-2), 129-39. [PubMed: 11000485]

(9). Moosmang S, Stieber J, Zong X, Biel M, Hofmann F, and Ludwig A (2001) Cellular expression and functional characterization of four hyperpolarization-activated pacemaker channels in cardiac and neuronal tissues. Eur. J. Biochem 268 (6), 1646-1652. [PubMed: 11248683]

(10). Shi W, Wymore R, Yu H, Wu J, Wymore RT, Pan Z, Robinson RB, Dixon JE, McKinnon D, and Cohen IS (1999) Distribution and prevalence of hyperpolarization-activated cation channel (HCN) mRNA expression in cardiac tissues. Circ. Res 85 (1), e1-e6. [PubMed: 10400919]

(11). George MS, Abbott LF, and Siegelbaum SA (2009) HCN hyperpolarization-activated cation channels inhibit EPSPs by interactions with M-type K(+) channels. Nat. Neurosci 12 (5), 577-84. [PubMed: 19363490]

(12). Magee JC (1999) Dendritic Ih normalizes temporal summation in hippocampal CA1 neurons. Nat. Neurosci 2 (9), 848.

(13). Cangiano L, Gargini C, Della Santina L, Demontis GC, and Cervetto L (2007) High-pass filtering of input signals by the Ih current in a non-spiking neuron, the retinal rod bipolar cell. PLoS One 2 (12), No. e1327. [PubMed: 18091997]

(14). Oswald MJ, Oorschot DE, Schulz JM, Lipski J, and Reynolds JN (2009) IH current generates the afterhyperpolarisation following activation of subthreshold cortical synaptic inputs to striatal cholinergic interneurons. J. Physiol 587 (24), 5879-5897. [PubMed: 19884321]

(15). Kase D, and Imoto K (2012) The Role of HCN Channels on Membrane Excitability in the Nervous System. J. Signal Transduction 2012, 619747.

(16). Nolan MF, Malleret G, Lee KH, Gibbs E, Dudman JT, Santoro B, Yin D, Thompson RF, Siegelbaum SA, Kandel ER, and Morozov A (2003) The hyperpolarization-activated HCN1 
channel is important for motor learning and neuronal integration by cerebellar Purkinje cells. Cell 115 (5), 551-64. [PubMed: 14651847]

(17). Nolan MF, Malleret G, Dudman JT, Buhl DL, Santoro B, Gibbs E, Vronskaya S, Buzsaki G, Siegelbaum SA, Kandel ER, and Morozov A (2004) A behavioral role for dendritic integration: HCN1 channels constrain spatial memory and plasticity at inputs to distal dendrites of CA1 pyramidal neurons. Cell 119 (5), 719-32. [PubMed: 15550252]

(18). Emery EC, Young GT, Berrocoso EM, Chen L, and McNaughton PA (2011) HCN2 ion channels play a central role in inflammatory and neuropathic pain. Science 333 (6048), 1462-6. [PubMed: 21903816]

(19). Wu S, Vysotskaya ZV, Xu X, Xie C, Liu Q, and Zhou L (2011) State-dependent cAMP binding to functioning HCN channels studied by patch-clamp fluorometry. Biophys. J 100 (5), 1226-32. [PubMed: 21354395]

(20). Wu S, Gao W, Xie C, Xu X, Vorvis C, Marni F, Hackett AR, Liu Q, and Zhou L (2012) Inner activation gate in S6 contributes to the state-dependent binding of cAMP in full-length HCN2 channel. J. Gen. Physiol 140 (1), 29-39. [PubMed: 22689828]

(21). Gao W, Su Z, Liu Q, and Zhou L (2014) State-dependent and site-directed photodynamic transformation of HCN2 channel by singlet oxygen. J. Gen. Physiol 143 (5), 633-44. [PubMed: 24733837]

(22). Proenza C, Angoli D, Agranovich E, Macri V, and Accili EA (2002) Pacemaker channels produce an instantaneous current. J. Biol. Chem 277 (7), 5101-9. [PubMed: 11741901]

(23). Mistrik P, Pfeifer A, and Biel M (2006) The enhancement of HCN channel instantaneous current facilitated by slow deactivation is regulated by intracellular chloride concentration. Pfluegers Arch 452 (6), 718-27. [PubMed: 16715293]

(24). Proenza C, and Yellen G (2006) Distinct populations of HCN pacemaker channels produce voltage-dependent and voltage-independent currents. J. Gen. Physiol 127 (2), 183-90. [PubMed: 16446506]

(25). Macri V, and Accili EA (2004) Structural elements of instantaneous and slow gating in hyperpolarization-activated cyclic nucleotide-gated channels. J. Biol. Chem 279 (16), 16832-46. [PubMed: 14752094]

(26). Zhu L, Wang X, Li XL, Towers A, Cao X, Wang P, Bowman R, Yang H, Goldstein J, Li YJ, and Jiang YH (2014) Epigenetic dysregulation of SHANK3 in brain tissues from individuals with autism spectrum disorders. Hum. Mol. Genet 23 (6), 1563-78. [PubMed: 24186872]

(27). Rosti RO, Sadek AA, Vaux KK, and Gleeson JG (2014) The genetic landscape of autism spectrum disorders. Dev. Med. Child Neurol 56 (1), 12-8. [PubMed: 24116704]

(28). Kouser M, Speed HE, Dewey CM, Reimers JM, Widman AJ, Gupta N, Liu S, Jaramillo TC, Bangash M, Xiao B, Worley PF, and Powell CM (2013) Loss of predominant Shank3 isoforms results in hippocampus-dependent impairments in behavior and synaptic transmission. $\mathrm{J}$. Neurosci 33 (47), 18448-68. [PubMed: 24259569]

(29). Peca J, Feliciano C, Ting JT, Wang W, Wells MF, Venkatraman TN, Lascola CD, Fu Z, and Feng G (2011) Shank3 mutant mice display autistic-like behaviours and striatal dysfunction. Nature 472 (7344), 437-42. [PubMed: 21423165]

(30). Bey AL, and Jiang YH (2014) Overview of mouse models of autism spectrum disorders. Curr. Protoc. Pharmacol 66 (5), 5.66.1-5.66.26. [PubMed: 25181011]

(31). Yi F, Danko T, Botelho SC, Patzke C, Pak C, Wernig M, and Sudhof TC (2016) Autismassociated SHANK3 haploinsufficiency causes Ih channelopathy in human neurons. Science 352 (6286), No. aaf2669. [PubMed: 26966193]

(32). Shcheglovitov A, Shcheglovitova O, Yazawa M, Portmann T, Shu R, Sebastiano V, Krawisz A, Froehlich W, Bernstein JA, Hallmayer JF, and Dolmetsch RE (2013) SHANK3 and IGF1 restore synaptic deficits in neurons from 22q13 deletion syndrome patients. Nature 503 (7475), 267-71. [PubMed: 24132240]

(33). Zhu M, Idikuda VK, Wang J, Wei F, Kumar V, Shah N, Waite CB, Liu Q, and Zhou L (2018) Shank3-deficient thalamocortical neurons show HCN channelopathy and alterations in intrinsic electrical properties. J. Physiol 596 (7), 1259-1276. [PubMed: 29327340] 
(34). Sherman SM (2016) Thalamus plays a central role in ongoing cortical functioning. Nat. Neurosci 19 (4), 533-41. [PubMed: 27021938]

(35). Sussman D, Leung RC, Vogan VM, Lee W, Trelle S, Lin S, Cassel DB, Chakravarty MM, Lerch JP, Anagnostou E, and Taylor MJ (2015) The autism puzzle: Diffuse but not pervasive neuroanatomical abnormalities in children with ASD. Neuroimage Clin 8, 170-9. [PubMed: 26106541]

(36). Nair A, Treiber JM, Shukla DK, Shih P, and Muller RA (2013) Impaired thalamocortical connectivity in autism spectrum disorder: a study of functional and anatomical connectivity. Brain 136 (6), 1942-1955. [PubMed: 23739917]

(37). Ludwig A, Budde T, Stieber J, Moosmang S, Wahl C, Holthoff K, Langebartels A, Wotjak C, Munsch T, Zong X, Feil S, Feil R, Lancel M, Chien KR, Konnerth A, Pape HC, Biel M, and Hofmann F (2003) Absence epilepsy and sinus dysrhythmia in mice lacking the pacemaker channel HCN2. EMBO J 22 (2), 216-24. [PubMed: 12514127]

(38). Abbas SY, Ying SW, and Goldstein PA (2006) Compartmental distribution of hyperpolarizationactivated cyclic-nucleotide-gated channel 2 and hyperpolarization-activated cyclic-nucleotidegated channel 4 in thalamic reticular and thalamocortical relay neurons. Neuroscience 141 (4), 1811-25. [PubMed: 16806719]

(39). Notomi T, and Shigemoto R (2004) Immunohistochemical localization of Ih channel subunits, HCN1-4, in the rat brain. J. Comp. Neurol 471 (3), 241-76. [PubMed: 14991560]

(40). Shah MM (2014) Cortical HCN channels: function, trafficking and plasticity. J. Physiol 592 (13), 2711-9. [PubMed: 24756635]

(41). Elinder F, Mannikko R, Pandey S, and Larsson HP (2006) Mode shifts in the voltage gating of the mouse and human HCN2 and HCN4 channels. J. Physiol 575 (2), 417-431. [PubMed: 16777944]

(42). Magee JC (1998) Dendritic hyperpolarization-activated currents modify the integrative properties of hippocampal CA1 pyramidal neurons. J. Neurosci 18 (19), 7613-24. [PubMed: 9742133]

(43). Nakamura Y, Shi XY, Numata T, Mori Y, Inoue R, Lossin C, Baram TZ, and Hirose S (2013) Novel HCN2 Mutation Contributes to Febrile Seizures by Shifting the Channel's Kinetics in a Temperature-Dependent Manner. PLoS One 8 (12), e80376. [PubMed: 24324597]

(44). Ying SW, Jia F, Abbas SY, Hofmann F, Ludwig A, and Goldstein PA (2007) Dendritic HCN2 channels constrain glutamate-driven excitability in reticular thalamic neurons. J. Neurosci 27 (32), 8719-32. [PubMed: 17687049]

(45). Chieng B, Azriel Y, Mohammadi S, and Christie MJ (2011) Distinct cellular properties of identified dopaminergic and GABAergic neurons in the mouse ventral tegmental area. J. Physiol 589 (15), 3775-3787. [PubMed: 21646409]

(46). Merrill CB, Friend LN, Newton ST, Hopkins ZH, and Edwards JG (2015) Ventral tegmental area dopamine and GABA neurons: Physiological properties and expression of mRNA for endocannabinoid biosynthetic elements. Sci. Rep 5, 16176. [PubMed: 26553597]

(47). Ford CP, Mark GP, and Williams JT (2006) Properties and opioid inhibition of mesolimbic dopamine neurons vary according to target location. J. Neurosci 26 (10), 2788-97. [PubMed: 16525058] 

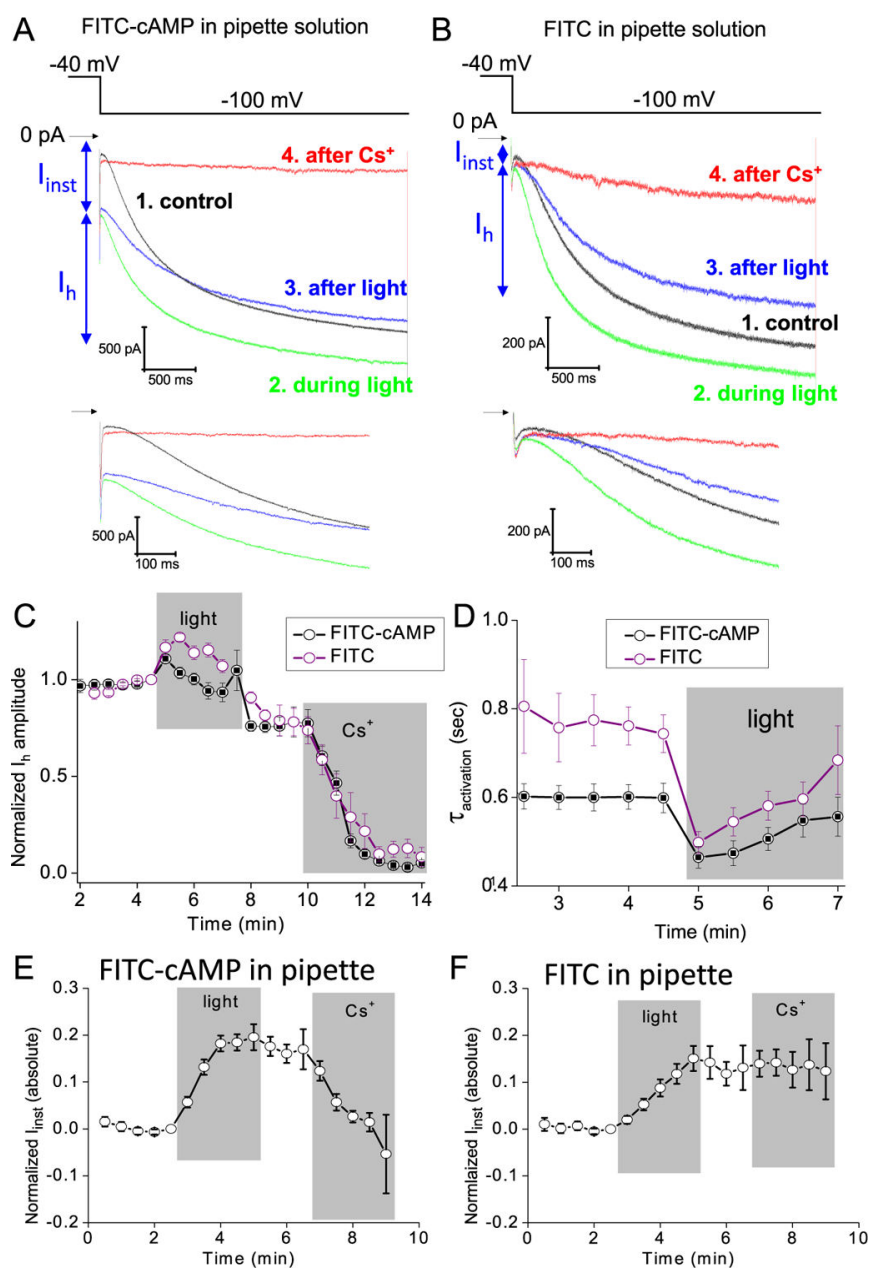

Figure 1.

Light illumination of TC neurons loaded with FITC-cAMP but not FITC alone results in an increase in $I_{\text {inst }}$ (A) FITC-cAMP $(20 \mu \mathrm{M})$ was delivered through the recording pipette. Top: Representative current traces recorded from a single $\mathrm{TC}$ neuron in response to a hyperpolarizing voltage step from -40 to $-100 \mathrm{mV}$. HCN currents were isolated using a cocktail of ion channel blockers (see Methods). $\mathrm{Cs}^{+}(1 \mathrm{mM})$ was applied near the end of the experiments to confirm the involvement of HCN channel. Light pulses were delivered to the brain slice through the objective lens. The control trace (in black) shows the last trace before the application of light. A total of five traces were recorded with light, and the last one is shown in green. Figure S1 shows all traces before, during, and after light for this data set. A zoomed-out view over the beginning of the hyperpolarization step is shown below. (B) FITC $(20 \mu \mathrm{M})$ was delivered through the recording pipette. The control trace (in black) shows the last trace before the application of light. A total of five traces were recorded with light, and the last one is shown in green. Figure S2 shows all traces before, during, and after light for this data set. A zoomed-out view over the beginning of the hyperpolarization step is shown below. (C) Time course of normalized $I_{\mathrm{h}}$ (normalize to the last current trace before light). Black: FITC-cAMP in pipette solution. Purple: FITC alone in pipette solution. (D) Averaged time constant of $I_{\mathrm{h}}$ current activation (fitted by a single-exponential function). (E) Time course of absolute $I_{\text {inst }}$ (relative to zero current) normalized to the $I_{\text {inst }}$ and $I_{\mathrm{h}}$ of the last trace 
before light: $\left(I_{\text {inst }}-I_{\text {inst,beforelight }}\right) / \mathrm{I}_{\mathrm{h}, \text { beforelight }}$. FITC-cAMP in pipette solution $(N=14)$. (F) Time course of normalized $I_{\text {inst }}$ (see previous equation) with FITC in pipette solution $(N=$ $8)$. 


\section{A FITC-CAMP in pipette solution}
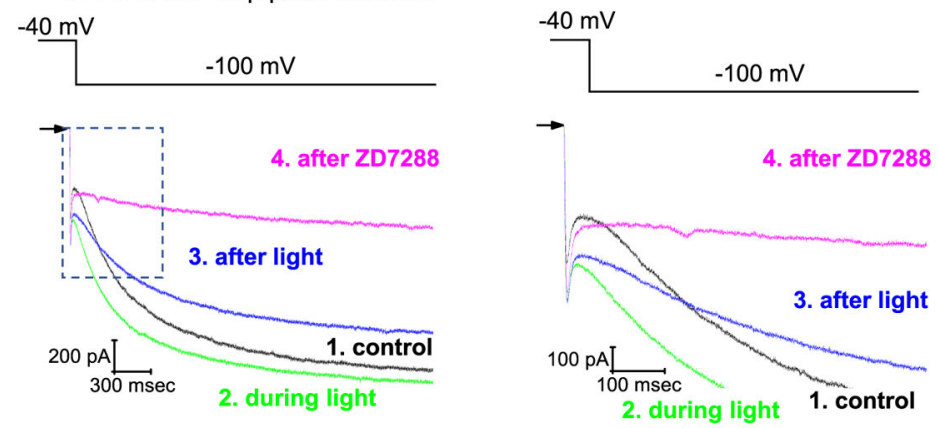

B

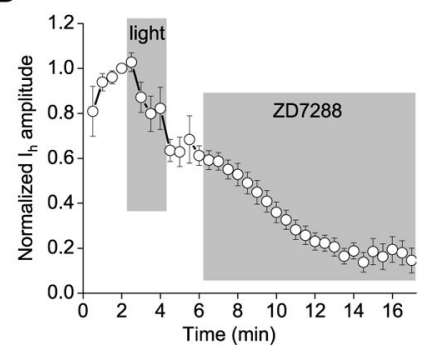

C

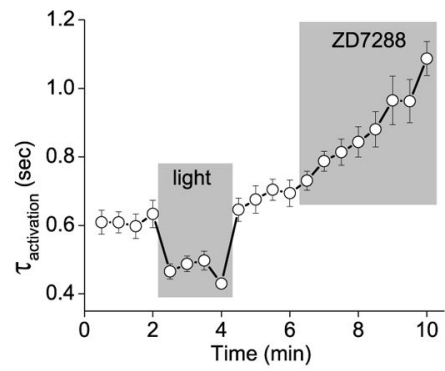

D

$\mathrm{E}$
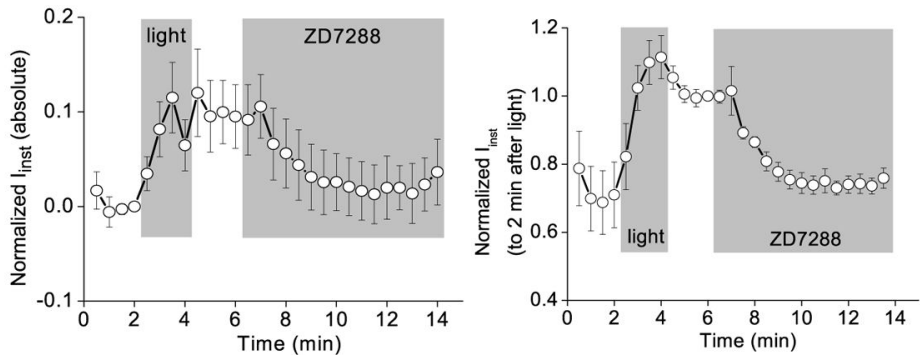

Figure 2.

PDM augmentation of $I_{\mathrm{h}}$ and $I_{\text {inst }}$ can be blocked by ZD7288. (A) Representative current traces recorded from a single TC neuron loaded with FITC-cAMP. ZD7288 was applied near the end of the experiments to confirm the contribution by $\mathrm{HCN}$ channels. A zoomed view over the box region is shown in the right. (B) Time course of normalized $I_{\mathrm{h}}$ (normalize to the $I_{\mathrm{h}}$ amplitude of the last trace before light) $(N=9)$. (C) Time constant of hyperpolarizationdependent channel activation. (D) Time course of absolute $I_{\text {inst }}$ (relative to zero current) normalized to the $I_{\text {inst }}$ and $I_{\mathrm{h}}$ of the last trace before light: ( $\left.I_{\text {inst }}-I_{\text {inst, before light }}\right) /$ $I_{\text {h, before light }}$ (E) Time course of absolute $I_{\text {inst }}$ (relative to zero current) normalized to the $I_{\text {inst }}$ of the last trace before ZD7288 application: $I_{\text {inst }} / I_{\text {inst, }}$ before ZD7288. 
A

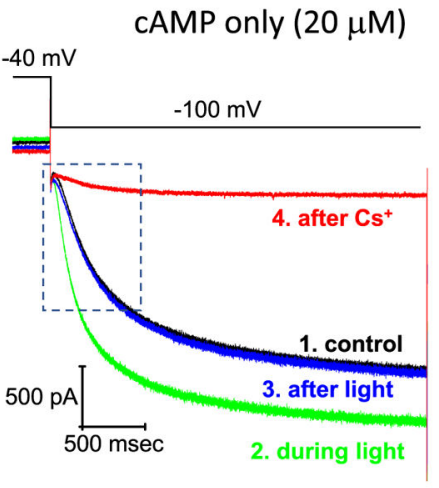

B

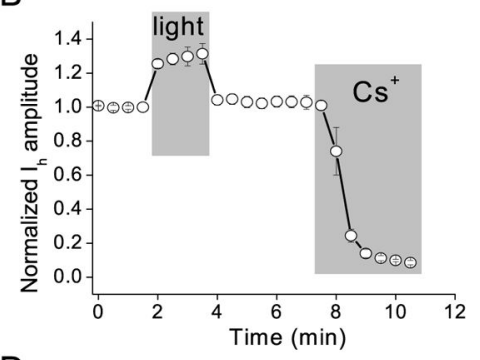

D

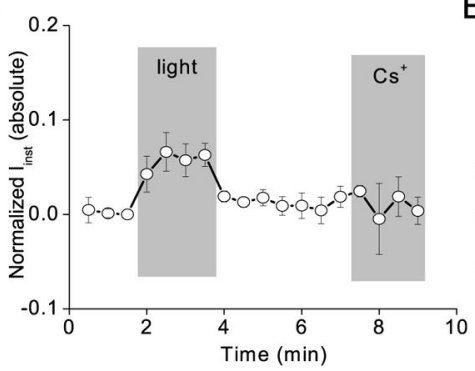

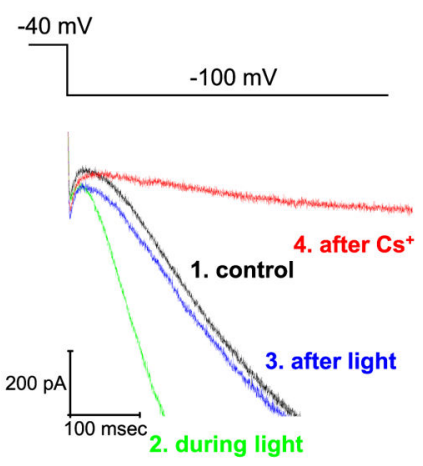

C

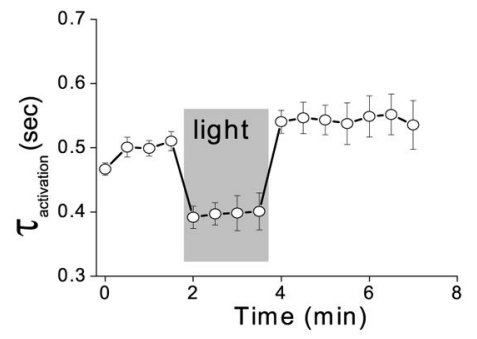

$\mathrm{E}$

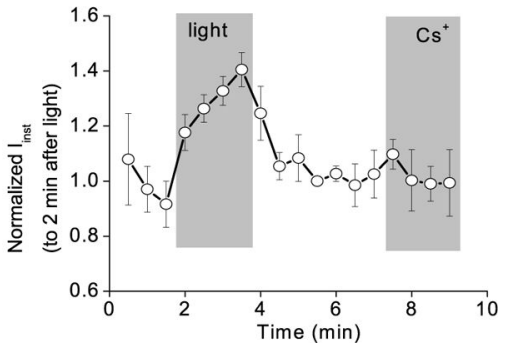

Figure 3.

Light illumination of TC neurons loaded with cAMP alone did not result in increases in $I_{\text {inst }}$. (A) cAMP $(20 \mu \mathrm{M})$ was delivered through the recording pipette. Representative current traces recorded from a single TC neuron in response to a hyperpolarizing voltage step from -40 to $-100 \mathrm{mV}$. The control trace (in black) shows the last trace before the application of light. A total of five traces were recorded with light, and the first one is shown in green. A zoomed view over the box region is shown on the right. (B) Time course of normalized $I_{\mathrm{h}}$ (normalized to the last trace before light) blocked by $\mathrm{Cs}^{+}(N=10)$. (C) Time constant of $I_{\mathrm{h}}$ current activation with cAMP added to the pipette solution. (D) Time course of absolute $I_{\text {inst }}$ (relative to zero current) normalized to the $I_{\text {inst }}$ and $I_{\mathrm{h}}$ of the last trace before light exposure: $\left(I_{\text {inst }}-I_{\text {inst, before light }}\right) / I_{\text {h, before light }}$ (E) Time course of absolute $I_{\text {inst }}$ (relative to zero current) normalized to the $I_{\text {inst }}$ of the last trace before $\mathrm{Cs}^{+}$application: $I_{\text {inst }} / I_{\text {inst, before } \mathrm{Cs}^{+}}$. 
A

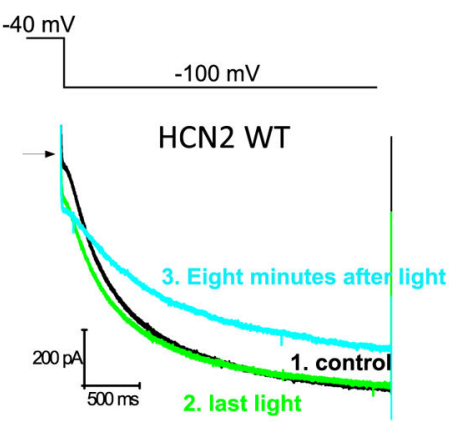

C

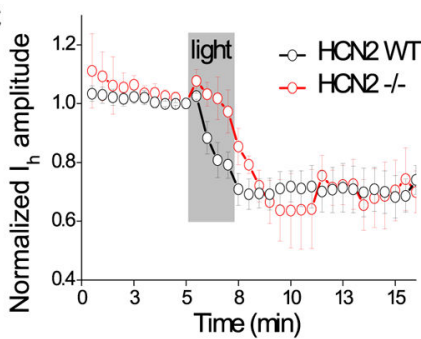

$\mathrm{E}$

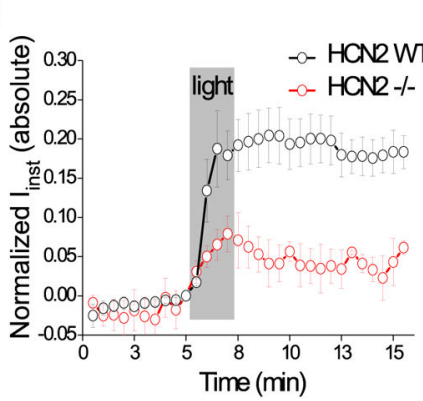

B

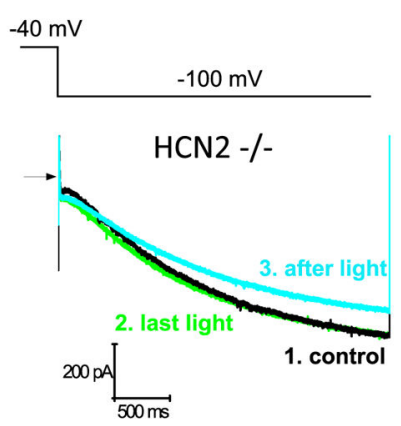

D 5 - HCNDWT

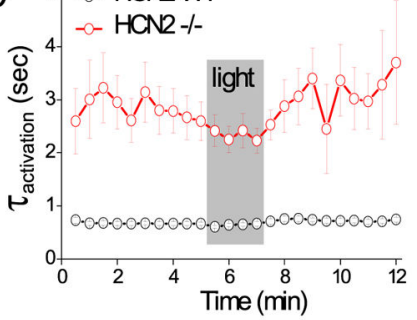

$\mathrm{F}$

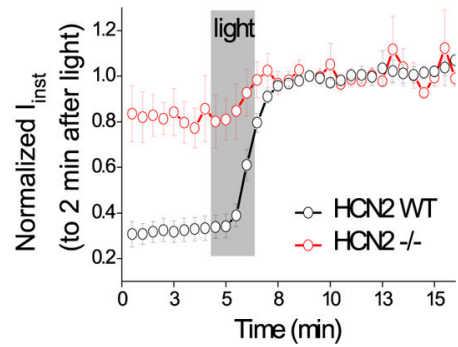

Figure 4.

PDM of HCN2-/- neurons resulted in minimal increases in $I_{\text {inst }}$ compared to WT neurons. (A) Representative current traces recorded from a WT neuron (right) loaded with FITCcAMP. Three current traces are shown: control before light (black), last trace with light (green), and 8 min after light (cyan). (B) Representative current traces recording from a HCN2-I- neuron. (C) Time course of normalized $I_{\mathrm{h}}$ (normalize to the $I_{\mathrm{h}}$ amplitude of the last trace before light pulse). FITC-cAMP $(20 \mu \mathrm{M})$ was added to the pipette solution (WT, $N$ $=7$; HCN2-l-, $N=4$ ). (D) Time constant of $I_{\mathrm{h}}$ current activation. (E) Time course of absolute $I_{\text {inst }}$ (relative to zero current) normalized to the $I_{\text {inst }}$ and $I_{\mathrm{h}}$ of the last trace before light: $\left(I_{\text {inst }}-I_{\text {inst, before light }}\right) / I_{\text {h, before light }}$. $(\mathrm{F})$ Time course of absolute $I_{\text {inst }}$ (relative to zero current) normalized to the $I_{\text {inst }}$ of the fourth trace after light: $I_{\text {inst }} / I_{\text {inst,fourth after light }}$. 
A
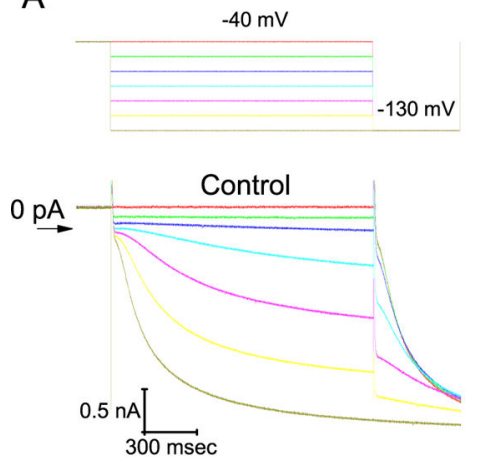

C

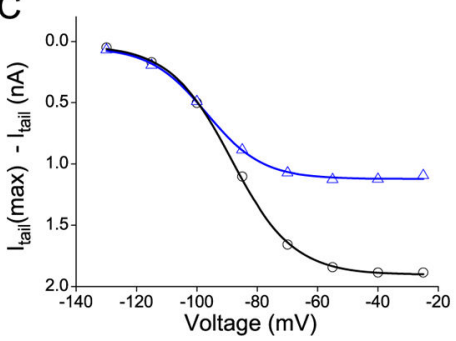

E

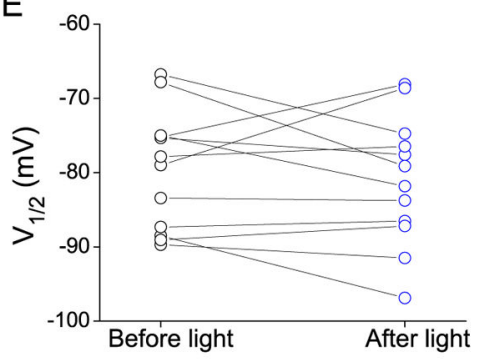

B
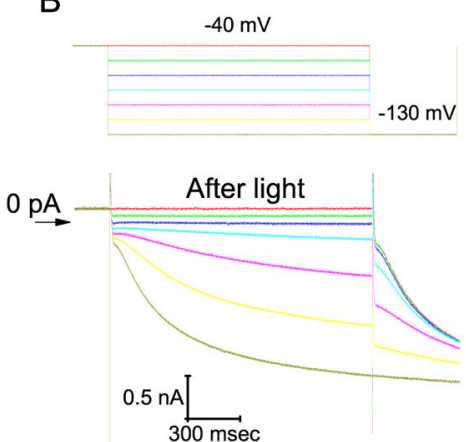

D

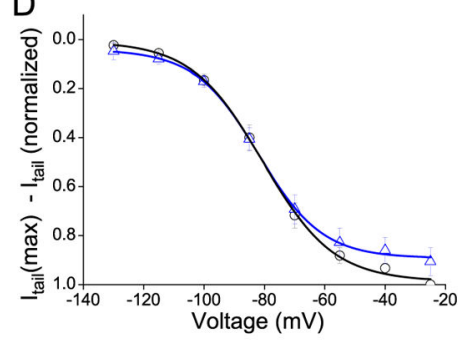

F

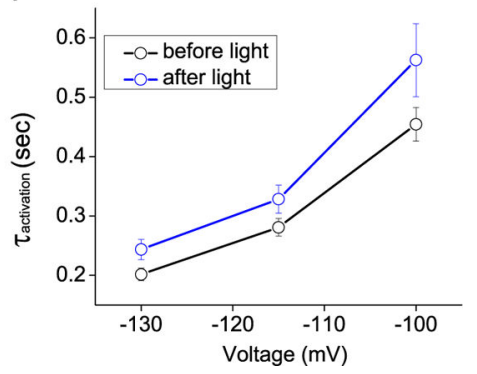

Figure 5.

PDM does not lead to significant changes to voltage-dependent gating of HCN channel. (A) Top: A series of hyperpolarizing voltage steps (15 mV steps) were used for channel activation. The tail current was measured at $-130 \mathrm{mV}$. Bottom: Representative current traces of a single TC neuron. (B) Results of the same neuron after application of light illumination. (C) Current-voltage relationship for the current traces shown in (A) and (B). Black, control before light. Blue, after light. (D) Averaged $I-V$ curves before (black) and after (blue) light pulses $(N=11)$. All data points were normalized to the increase in tail current amplitude (beginning of the $-130 \mathrm{mV}$ step to the end of the $-130 \mathrm{mV}$ ) measured at the voltage step of $-25 \mathrm{mV}$. (E) Summary of $V_{1 / 2}$ values for each cell before (black) and after light pulses (blue). (F) Time constant of activation of $I_{\mathrm{h}}$ current vs voltage steps $(N=12)$. 
A

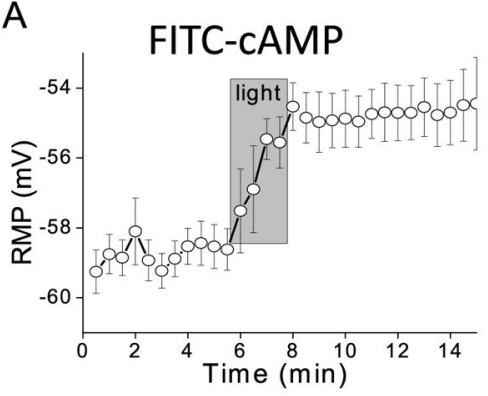

C

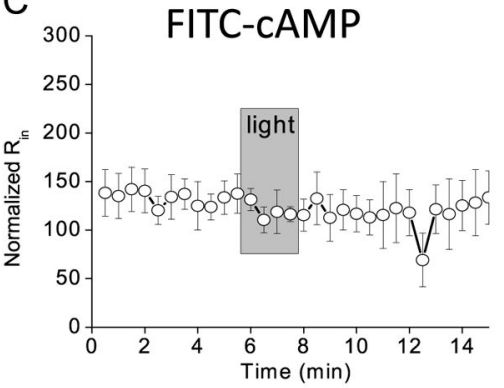

E

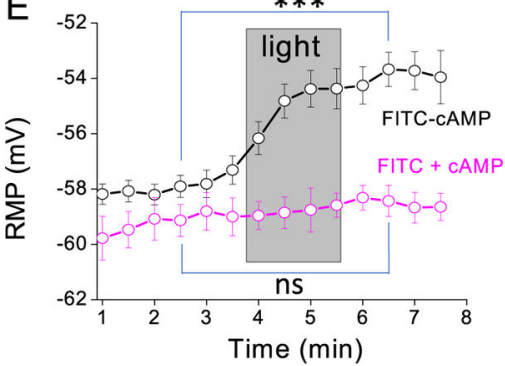

B

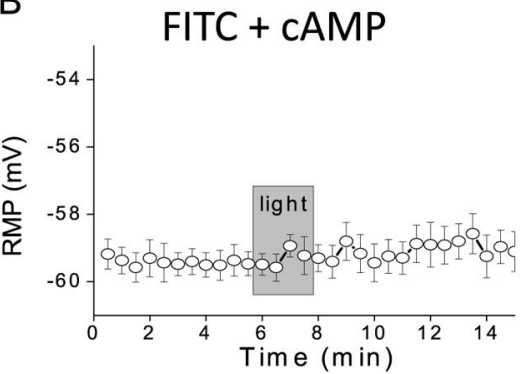

D $\quad$ FITC + CAMP

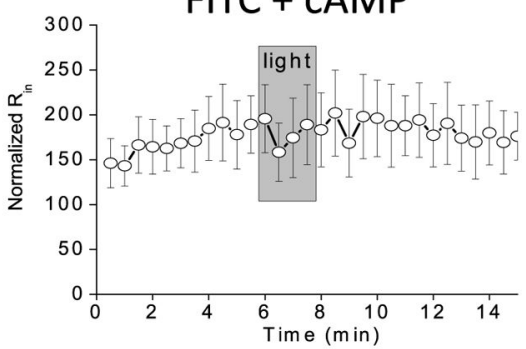

$\mathrm{F}$

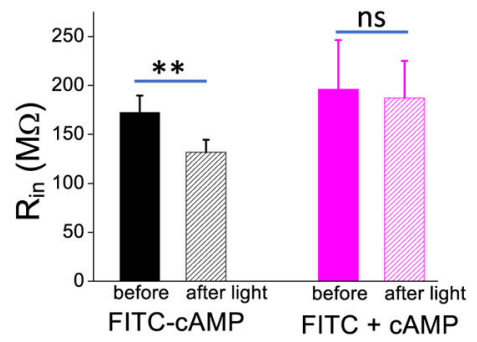

Figure 6.

Effects of light illumination on RMP and $R_{\text {in }}$ of neurons loaded with FITC-cAMP or with FITC + cAMP. Two slightly different protocols were used in these experiments. For (A-D), a current injection of $+10 \mathrm{pA}$ was used for the measurement of $R_{\text {in }}$ (as well as RMP) of all traces. For $6 \mathrm{E}-6 \mathrm{~F}, R_{\text {in }}$ was measured twice, before and after light illumination, respectively, whereas RMP was measured for every trace before a current injection of $-100 \mathrm{pA}$. (A) Time course of RMP with FITC-cAMP loaded into TC neurons through the pipette solution. Light pulses were applied during the traces indicated by the gray box. Averaged RMP, before light, $-58.8 \pm 0.6 \mathrm{mV}$; after light, $-55.6 \pm 0.5 \mathrm{mV} ; N=5 ; P<0.001$ (paired $t$ test). (B) Time course of changes in RMP with FITC + cAMP (two separate chemicals added together into the pipette solution). Averaged RMP, before light, RMP $=-59.5 \pm 0.4 \mathrm{mV}$; after light, -59.2 $\pm 0.5 \mathrm{mV} ; N=6 ; P=0.65$ (paired $t$ test). (C) Time course of changes in $R_{\text {in }}$ with FITCcAMP in the pipette solution. Averaged $R_{\mathrm{in}}$, before light, $129.1 \pm 18.6 \mathrm{M} \Omega$; after light, 120.7 $\pm 21.5 \mathrm{M} \Omega ; N=5 ; P=0.07$ (paired $t$ test). (D) Time course of changes in $R_{\mathrm{in}}$ with FITC + cAMP in the pipette solution. Averaged $R_{\mathrm{in}}$, before light, $166.5 \pm 28.3 \mathrm{M} \Omega$; after light, 192.5 $\pm 41.6 \mathrm{M} \Omega ; N=6 ; P=0.17$ (paired $t$ test). (E) Time course of changes in RMP. Black, FITC-cAMP in pipette solution. Purple, FITC + cAMP in pipette solution. Averaged RMP, before light, $-58.1 \pm 0.4 \mathrm{mV}$; after light, $-54.1 \pm 0.6 \mathrm{mV} ; N=9 ; P<0.001$ (paired $t$ test; *** in the figure). FITC + cAMP, before light, $-59.2 \pm 0.6 \mathrm{mV}$; after light, $-58.5 \pm 0.5 \mathrm{mV} ; N=$ 
4; $P=0.21$ (paired $t$ test; ns in the figure). Light pulses were applied for $2 \mathrm{~s}$ for the traces in the gray box. (F) Averaged $R_{\text {in }}$. FITC-cAMP in pipette solution, before light, $172.4 \pm 17.3$ $\mathrm{M} \Omega$; after light, $131.8 \pm 12.6 \mathrm{M} \Omega ; N=6 ; P=0.0025$ (paired $t$ test; $* *$ in the figure). FITC + cAMP in pipette solution, before light, $196.2 \pm 50.1 \mathrm{M} \Omega$; after light, $187.3 \pm 37.8 \mathrm{M} \Omega ; N=$ 3; $P>0.05$ (paired $t$ test; ns in the figure). 

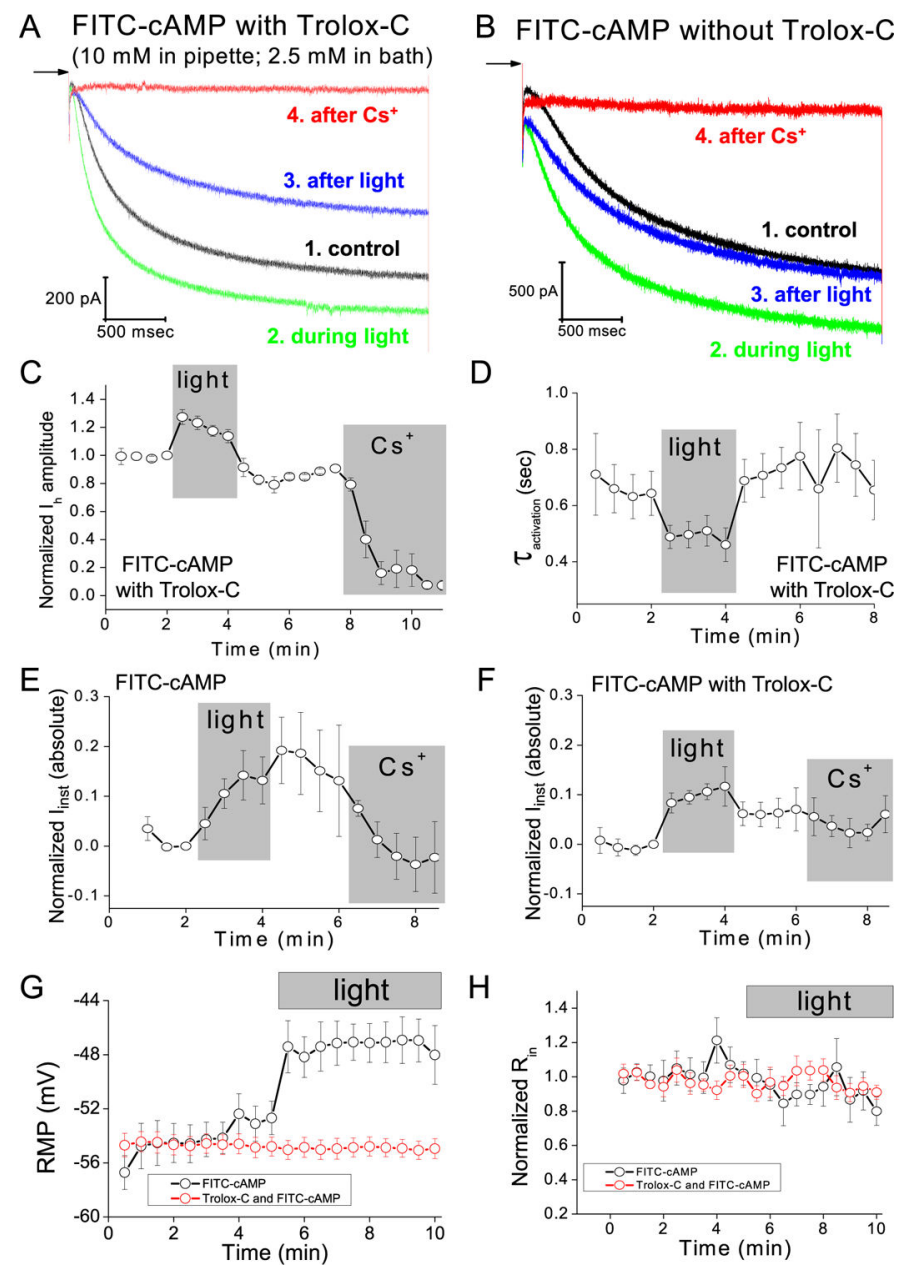

Figure 7.

Trolox-C, a quencher for ${ }^{1} \mathrm{O}_{2}$, abolished the increase in $I_{\text {inst }}$ and the changes in RMP and $R_{\text {in }}$ following light pulses. (A) Current traces recorded with Trolox-C added to the pipette solution (10 mM; with $20 \mu \mathrm{M}$ FITC-cAMP) and bath solution $(2.5 \mathrm{mM})$. (B) Current traces recorded with FITC-cAMP $(20 \mu \mathrm{M})$ but without Trolox-C as the positive control for the increase in $I_{\text {inst }}$ after PDM. (C) Time course of $I_{\mathrm{h}}$ amplitude normalized to the last trace before light $(N=8)$. (D) Time course of time constant of $I_{\mathrm{h}}$ current activation $(N=8)$. (E) FITC-cAMP without Trolox-C. Time course of absolute $I_{\text {inst }}$ (relative to zero current) normalized to the $I_{\text {inst }}$ and $I_{\mathrm{h}}$ of the last trace before light: $\left(I_{\text {inst }}-I_{\text {inst, before light }}\right) /$ $I_{\mathrm{h}}$, before light. (F) FITC-cAMP with Trolox-C. Time course of absolute $I_{\text {inst }}$ (relative to zero current) normalized to the $I_{\text {inst }}$ and $I_{\mathrm{h}}$ of the last trace before light: ( $I_{\text {inst }}-I_{\text {inst, before }}$ light $) /$ $I_{\mathrm{h}}$, before light $(\mathrm{G})$ Time course of RMP without (black; before light, $-54.4 \pm 1.3 \mathrm{mV}, N=8$; after light, $-47.3 \pm 1.5 \mathrm{mV}, N=8 ; P<0.01$ ) or with (red; before light, $-55.8 \pm 0.9 \mathrm{mV}, N=$ 11; after light, $-55.1 \pm 0.8 \mathrm{mV}, N=11, P=0.20$ ) Trolox-C. (H) Time course of $R_{\text {in }}$ without (black; before light, $234.5 \pm 48.8 \mathrm{M} \Omega, N=8$; after light, $226.3 \pm 48.6 \mathrm{M} \Omega, N=8, P=0.64$ ) or with (red; before light, $185.7 \pm 37.0 \mathrm{M} \Omega, N=11$; after light, $174.5 \pm 28.0 \mathrm{M} \Omega, N=11, P$ $=0.53$ ) Trolox $-\mathrm{C}$. 
A

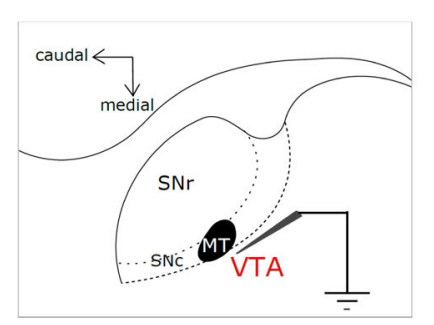

C

FITC-cAMP in pipette solution

$n$

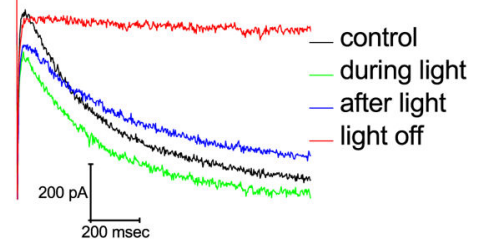

$\mathrm{E}$
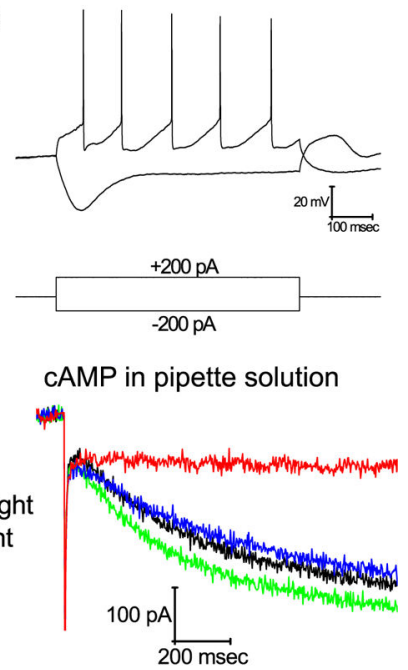

cAMP in pipette solution
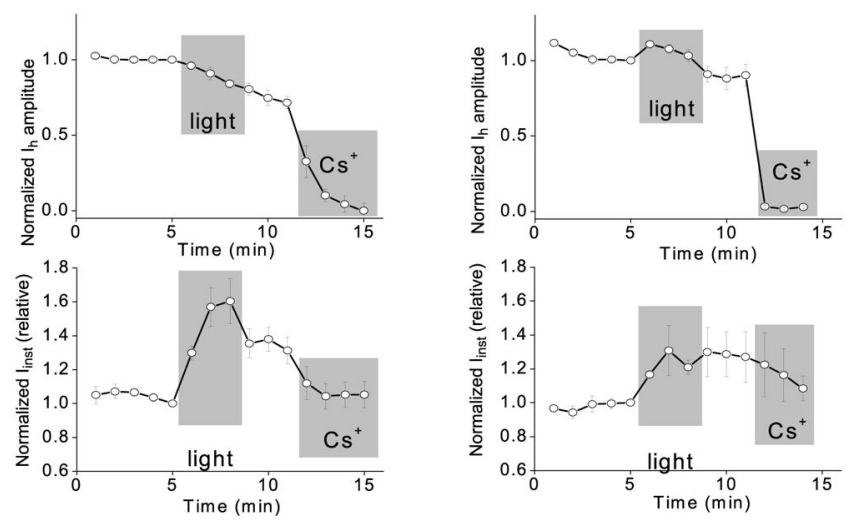

Figure 8.

PDM of native HCN channels expressed in VTA neurons. (A) Schematic drawing showing the location of dopaminergic cell bodies in the VTA. (B) Typical action potential firing and membrane potential "sag" in response to $+200 \mathrm{pA}$ (top) and $-200 \mathrm{pA}$ (bottom) current injections. (C) Representative current traces recorded from a VTA neuron loaded with FITCcAMP $(20 \mu \mathrm{M})$. Black, before light; green, light; blue, after light; red, after light with $\mathrm{Cs}^{+}$. (D) Averaged results of neurons loaded with FITC-cAMP. Top: Normalized amplitude of the $I_{\mathrm{h}}$ component. Bottom: Amplitude of the $I_{\text {inst }}$ component (normalized to the last trace before light) $(N=12)$. (E) Representative current traces recorded from a VTA neuron loaded with cAMP $(20 \mu \mathrm{M})$. Black, before light; green, light; blue, after light; red, after light with $\mathrm{Cs}^{+}$. (F) Averaged results of VTA neurons loaded with cAMP. Top: Normalized amplitude of the $I_{\mathrm{h}}$ component after light and application of $\mathrm{Cs}^{+}$. Bottom: Amplitude of the $I_{\text {inst }}$ component (normalized to the last trace before light) after light and application of $\mathrm{Cs}^{+}(N=9)$. 